\title{
High Tibial Osteotomy for Medial Knee Osteoarthritis
}

\author{
Hee-Soo Kyung, $\mathrm{MD}, \mathrm{PhD}$
}

Department of Orthopedic Surgery, School of Medicine, Kyungpook National University, Daegu, Korea

High tibial osteotomy (HTO) has been rapidly adopted as a treatment for medial knee osteoarthritis with varus deformity ${ }^{1,2)}$. This lower extremity realignment procedure is used to relieve pain and correct varus deformity of the knee joint. Preoperative planning to determine the correction gap and angle has a significant effect on postoperative results of HTO. However, postoperative correction can be incongruent with the preoperative plan, even with the use of a navigation system and computer imaging. In a clinical situation, postoperative correction may be influenced by several factors, such as muscle action in walking and ligament balance ${ }^{3)}$. Still, the main purpose of HTO is relief of pain caused by osteoarthritis, rather than correction of the deformity, and most patients have shown improvement in clinical symptoms. However, good long-term results can be expected if accurate correction can be achieved through careful preoperative planning ${ }^{4}$.

Traditionally, the cable method using a radiopaque line or a metal rod has been popular for the determination of correction in HTO as it allows real-time monitoring of the mechanical axis during surgery; however, the results can deviate due to nonweight bearing status and the influence of limb rotation during osteotomy, and it can increase radiation exposure during evaluation of the hip and ankle centers. Accordingly, preoperative planning using full-length weight bearing lower limb radiographs has recently been introduced for calculation of the correction angle and gap in weight bearing status using a picture archiving and communication system (PACS) or special software ${ }^{5,6)}$. In this is-

Correspondence to: Hee-Soo Kyung, MD, PhD

Department of Orthopedic Surgery, School of Medicine, Kyungpook

National University, Daegu 41944, Korea

Tel: +82-53-200-5636, Fax: +82-53-422-6605

E-mail: hskyung@knu.ac.kr

This is an Open Access article distributed under the terms of the Creative Commons Attribution Non-Commercial License (http://creativecommons.org/licenses/by-nc/4.0/) which permits unrestricted non-commercial use, distribution, and reproduction in any medium, provided the original work is properly cited. sue, we present a report comparing the two methods; the report showed that the PACS method yielded more accurate results with less radiation exposure. Some surgeons have suggested that HTO using a navigation system results in an accurate correction angle ${ }^{7.8)}$. However, navigation-assisted HTO is performed in the non-weight bearing status, and thus the correction angle will change postoperatively in weight bearing status. In general, the Fujisawa point (a point $62.5 \%$ from the end of the medical tibial condyle) is considered the optimal location of the mechanical axis for deformity correction in $\mathrm{HTO}^{9)}$. In the meantime, there are interesting reports stating that determination of the correction angle in open wedge HTO should consider the mechanical axis of the contralateral knee for balanced alignment of the lower extremities.

This issue of Knee Surgery and Related Research contains a report on open wedge HTO combined with arthroscopic surgery, which emphasized intra-articular debridement. The abrasion of eroded cartilage and removal of cartilage debris improved mechanically-induced symptoms ${ }^{10,11)}$.

Usually, a donor site defect is neglected after autogenous iliac bone grafting. However, there have been some interesting reports, including a report by Lee et al. published in this issue, showing that reconstruction of the iliac crest with bone cement decreases donor site pain and morbidity after autogenous iliac bone grafting in open wedge HTO.

A metal plate is frequently used for HTO. There are several reports on complications related to the plate, including screw loosening, metal failure, loss of correction angle, and infection ${ }^{12,13)}$. However, Seo et al. reported that only minor complications were noted after HTO using a strong locking plate, and they concluded that the strong locking plate should be used for open wedge HTO due to many advantages it offers.

HTO is often performed in patients with medial knee osteoarthritis with varus deformity. Preoperative counseling with the patient is very important; the patient should be aware that the 
main purpose of HTO is pain relief, not complete resolution of osteoarthritis. The main complaints of the procedure including postoperative changes in the degree of correction in the lower extremity and leg length should also be taken into consider$\operatorname{ation}^{14,15)}$.

\section{References}

1. Dean CS, Liechti DJ, Chahla J, Moatshe G, LaPrade RF. Clinical outcomes of high tibial osteotomy for knee instability: a systematic review. Orthop J Sports Med. 2016;4: 2325967116633419.

2. Sabzevari S, Ebrahimpour A, Roudi MK, Kachooei AR. High tibial osteotomy: a systematic review and current concept. Arch Bone Jt Surg. 2016;4:204-12.

3. Ogawa H, Matsumoto K, Ogawa T, Takeuchi K, Akiyama H. Preoperative varus laxity correlates with overcorrection in medial opening wedge high tibial osteotomy. Arch Orthop Trauma Surg. 2016;136:1337-42.

4. Amendola A, Bonasia DE. Results of high tibial osteotomy: review of the literature. Int Orthop. 2010;34:155-60.

5. Schroter S, Ihle C, Mueller J, Lobenhoffer P, Stockle U, van Heerwaarden R. Digital planning of high tibial osteotomy. Interrater reliability by using two different software. Knee Surg Sports Traumatol Arthrosc. 2013;21:189-96.

6. Marx RG, Grimm P, Lillemoe KA, Robertson CM, Ayeni OR, Lyman S, Bogner EA, Pavlov H. Reliability of lower extremity alignment measurement using radiographs and PACS. Knee Surg Sports Traumatol Arthrosc. 2011;19:1693-8.

7. Akamatsu Y, Kobayashi H, Kusayama Y, Kumagai K, Saito T. Comparative study of opening-wedge high tibial osteotomy with and without a combined computed tomography-based and image-free navigation system. Arthroscopy. 2016;32:
2072-81.

8. Jones LD, Brown CP, Jackson W, Monk AP, Price AJ. Assessing accuracy requirements in high tibial osteotomy: a theoretical, computer-based model using AP radiographs. Knee Surg Sports Traumatol Arthrosc. 2016 Apr 7 [Epub]. http:// dx.doi.org/10.1007/s00167-016-4092-3.

9. Fujisawa Y, Masuhara K, Shiomi S. The effect of high tibial osteotomy on osteoarthritis of the knee: an arthroscopic study of 54 knee joints. Orthop Clin North Am. 1979;10:585608.

10. Rozkydal Z, Kura V, Ondrusek S. The arthroscopic debridement in the management of osteoarthritis of the knee joint by high tibial osteotomy. Bratisl Lek Listy. 2003;104:362-6.

11. Keene JS, Dyreby JR Jr. High tibial osteotomy in the treatment of osteoarthritis of the knee: the role of preoperative arthroscopy. J Bone Joint Surg Am. 1983;65:36-42.

12. Woodacre T, Ricketts M, Evans JT, Pavlou G, Schranz P, Hockings M, Toms A. Complications associated with opening wedge high tibial osteotomy: a review of the literature and of 15 years of experience. Knee. 2016;23:276-82.

13. Kyung HS, Lee BJ, Kim JW, Yoon SD. Biplanar open wedge high tibial osteotomy in the medial compartment osteoarthritis of the knee joint: comparison between the Aescula and TomoFix Plate. Clin Orthop Surg. 2015;7:185-90.

14. Kim JI, Kim BH, Lee KW, Lee O, Han HS, Lee S, Lee MC. Lower limb length discrepancy after high tibial osteotomy: prospective randomized controlled trial of lateral closing versus medial opening wedge osteotomy. Am J Sports Med. 2016 Aug 8 [Epub]. http://dx.doi.org/10.1177/03635465166 59284.

15. Loia MC, Vanni S, Rosso F, Bonasia DE, Bruzzone M, Dettoni F, Rossi R. High tibial osteotomy in varus knees: indications and limits. Joints. 2016;4:98-110. 